\section{NEONATAL MAGNETIC RESONANCE IMAGING IN EXTREMELY LOW GESTATIONAL AGE INFANTS AND OUTCOME AT 30 MONTHS}

\author{
B. Skiöld ${ }^{1}$, B. Vollmer ${ }^{1}$, B. Böhm¹, B. Hallberg², \\ S. Horsch ${ }^{2,3}$, M. Mosskin ${ }^{4}$, H. Lagercrantz ${ }^{1}$, \\ U. Ådén ${ }^{1}$, M. Blennow ${ }^{1}$ \\ ${ }^{1}$ Dept of Women's and Children's Health, \\ Karolinska Institutet, ${ }^{2}$ Inst CLINTEC, Karolinska \\ Institute, Stockholm, Sweden, ${ }^{3}$ Dept of \\ Neonatology, Erasmus MC/ Sophia Children's \\ Hospital, Rotterdam, The Netherlands, ${ }^{4}$ Dept of \\ Neuroradiology, Karolinska University Hospital, \\ Stockholm, Sweden
}

Aim: To examine associations of structural brain abnormalities, in particular diffuse excessive high signal intensities (DEHSI), assessed by visual inspection of neonatal magnetic resonance images (MRI) with neurodevelopmental outcome at age 30 months corrected in a 3 year cohort of extremely low gestational age (ELGA) infants.

Methods: 109 infants born < 27 weeks of gestation underwent conventional $\mathrm{MRI}$ at term-equivalent age. Images were analysed using an established scoring system (Inder et al 2003). At age 30 months corrected the infants were assessed using the Bayley Scales of Infant and Toddler Development.

Results: No or mild white matter abnormalities (WMA) were seen in $86 \%$ of infants. In $14 \%$ moderate or severe WMA, and in $5 \%$ grey matter abnormalitites (GMA) were detected. DEHSI were seen in $56 \%$. Outcome data were available for $72 \%$ of the cohort. Mean composite scores were 95 (SD \pm 10$)$ for cognitive scales, $97(S D \pm 14)$ for language scales, and $103(\mathrm{SD} \pm 14)$ for motor scales. Six percent of the infants had cerebral palsy. Significant associations were seen between moderate-severe WMA and/or GMA and cognitive scores (Mann-Whitney U-test, $p<0.05$ ) as well as language scores $(p<0.05)$. No significant associations were seen between MRI findings and motor scores. DEHSI were not associated with performance on the Bayley Scales.

Conclusion: In our cohort of ELGA infants, moderate-severe brain abnormalitites on neonatal MRI were associated with impaired cognitive and language outcome at age 30 months corrected. The incidence of DEHSI was high, but the presence of DEHSI was not associated with adverse outcome.

\section{COMPUTERIZED COGNITIVE TRAINING IMPROVES WORKING MEMORY AND \\ LEARNING IN EXTREMELY LOW BIRTH WEIGHT (ELBW) ADOLESCENTS}

G.C. Løhaugen ${ }^{1,2}$, I. Antonsen ${ }^{3}$, A. Gramstad ${ }^{4}$, T. Vik ${ }^{2}$, A. Håberg, $3,5,6$ A.-M. Brubakk ${ }^{2,7}$, J. Skranes ${ }^{1,2}$

${ }^{1}$ Dept of Pediatrics, Sørlandet Hospital, Arendal, 'Dept of Lab Medicine, Children's and Women's Health, ${ }^{3}$ Dept of Circulation and Medical Imaging, Norwegian University of Science and Technology,

Trondheim, ${ }^{4}$ Dept of Psychology, University

of Bergen, Bergen, ${ }^{5}$ Dept of Neuroscience, Norwegian University of Science and Technology,

${ }^{6}$ Dept of Diagnostic Imaging, MR Center, ${ }^{7}$ Dept

of Pediatrics, St Olav's University Hospital, Trondheim, Norway

Background and aims: Being born preterm with ELBW (birth weight $<1000 \mathrm{~g}$ ) constitutes an increased risk of learning disabilities and psychiatric symptoms, including attention deficit and working memory (WM) problems. WM is our ability to hold visual and/or verbal information online while manipulating it (i.e. doing mental arithmetic). Aim of study was to evaluate the effect of daily use of a computerized WM training program (Cogmed RM) in ELBW adolescents compared to controls. We assessed verbal and visual aspects of WM as well as generalizing effects on learning.

Methods: Sixteen ELBW and 19 controls age 1415 participated in a study from St.Olav's University Hospital in Trondelag, Norway. The control group consisted of term born children with normal birth weight. An extensive neuropsychological assessment was performed prior to, immediately after training, and at 6 months follow up. Participants used the Cogmed RM computer program at home for 30 minutes a day, 5 days a week in 5 weeks.

Results: Before training the ELBW subjects obtained lower IQ scores and lower scores on visual WM, as well on delayed memory for a verbal story. Both groups significantly improved their WM results on visual and verbal WM tasks, and obtained better results on non-trained tasks measuring learning ability for verbal material. After training there were no significant differences between groups on any outcome measurements. Six months follow up showed sustained effects of training. 
Conclusions: Computerized WM training may be an effective intervention to reduce learning and memory deficits in ELBW adolescents.

108

\section{THE COURSE OF APPARENT DIFFUSION COEFFICIENT AFTER PERINATAL ARTERIAL ISCHEMIC STROKE}

N.E. van der $A a^{1}$, M.J.N.L. Benders ${ }^{1}$, P. Anbeek ${ }^{2}$, K.L. Vincken ${ }^{2}$, F. Groenendaal ${ }^{1}$, L.S. de Vries ${ }^{1}$

${ }^{1}$ Department of Neonatology, ${ }^{2}$ Image Sciences Institue, UMC Utrecht, Utrecht, The Netherlands

Introduction: After a perinatal arterial ischemic stroke (PAIS), ischemic tissue can be clearly visualised using diffusion weighted imaging (DWI) and the derived apparent diffusion coefficient (ADC) map. Aim of this study was to quantify the course of $A D C$ values of the stroke throughout the first week.

Methods: Term born infants born between 2003 and 2010, whose PAIS was confirmed using DWI within the first week of life were included. A second scan was acquired at the age of three months and included an IR and T2 sequence.

Regions of interest were drawn on the ADC map of the first scan in the area which evolved to an area of cavitation on the second scan. Linear regression analysis was performed to assess the relationship between postnatal age (hours) and the ADC value.

Results: Forty-two infants were scanned. Territories involved included MCA main branch (10), MCA cortical branch (17), MCA lenticulostriatal (1), PCA (7) and watershed areas (7). Mean postnatal age at scan was $105 \mathrm{~h}$ (range: $57-167 \mathrm{~h}$ ). When analyzing the whole group the postnatal age correlated with the ADC value $\left(R^{2}=0.38, p<0.001\right)$. This correlation was also present in the MCA main and MCA cortical branches subgroups $\left(R^{2}=0.54, p=0.02\right.$ and $R^{2}=0.58, p<0.001$ respectively).

Conclusion: Between day 2 and 7 a significant correlation between postnatal age and ADC value of the stroke area was found in the core of the ischemic tissue. Although a small number of scans was used, this correlation might be useful to estimate the timing of the onset of stroke.
109

\section{EVALUATION OF DEXTROMETHORPHAN AS NEUROPROTECTIVE STRATEGY IN NEONATAL HYPEROXIC BRAIN INJURY}

E. Griesmaier ${ }^{1}$, C. Kampkötter ${ }^{2}$, A. Posod ${ }^{1}$, I. Bendix², M. Urbanek ${ }^{1}$, U. Kiechl-Kohlendorfer ${ }^{1}$, U. Felderhoff-Müser ${ }^{2}$, M. Keller ${ }^{2}$

${ }^{1}$ Department of Pediatrics IV, Medical University of Innsbruck, Innsbruck, Austria, ${ }^{2}$ Department of Pediatrics I, Neonatology, University Hospital of Essen, Essen, Germany

Background and aims: Hyperoxia is a high risk factor in the pathogenesis of preterm brain injury. Oxygen, at supraphysiological concentrations has been shown to cause an increase in free radical formation, inflammatory response and apoptotic cell death and subsequently contributes to brain injury. Dextromethorphan (DM) has been shown to be protective against inflammation-mediated, hypoxic-ischemic and excitotoxic brain injury. This study focuses on potential effects and underlying mechanisms of DM in a neonatal animal model of hyperoxic brain injury.

Methods: On postnatal day six (P6) rat pups were randomly injected intraperitoneally with i) DM $5 \mu \mathrm{g} / \mathrm{g}$ body weight, (bw), ii) DM $25 \mu \mathrm{g} / \mathrm{g}$ bw and iii) PBS as control. Subsequent to the injection rats were exposed to either normoxia $(21 \%$ O2) or hyperoxia $\left(>90 \% \mathrm{O}_{2}\right)$ for 24 hours. Endpoint was set at P7. Western Blot (WB) and immunohistochemistry analysed the effect of DM on apoptosis by activation of caspase-3 and on pro- and anti-inflammatory cytokines (interleukin (IL) 18 and 10).

Results: AsingledoseofDM $(25 \mu \mathrm{g} / \mathrm{g} \mathrm{bw})$ significantly reduced the number of caspase-3 positive cells after hyperoxia in temporoparietal, parietofrontal and retrosplenial cortex and in occipital and frontal white matter. Preliminary data indicate that IL-10 levels analysed by WB are decreased by hyperoxia and restored to normal levels by administration of DM. DM showed no effect on interleukin 18 expression.

Conclusion: This study shows a protective effect of DM against hyperoxia-induced apoptotic cell death and inflammatory responses in the newborn rat brain. 